BMJ Open Sport \& Exercise Medicine

\section{Exploring the association between recent concussion, subconcussive impacts and depressive symptoms in male Australian Football players}

To cite: Harris SA, Chivers PT, Mclntyre FL, et al. Exploring the association between recent concussion, subconcussive impacts and depressive symptoms in male Australian Football players. BMJ Open Sport \& Exercise Medicine 2020;6:e000655. doi:10.1136/ bmjsem-2019-000655

- Additional material is published online only. To view please visit the journal online (http://dx.doi.org/10.1136/ bmjsem-2019-000655).

This work was previously presented at the ASICS Sports Medicine Australia 2017 National Conference in Malaysia.

Accepted 7 February 2020

Check for updates

(c) Author(s) (or their employer(s)) 2020. Re-use permitted under CC BY-NC. No commercial re-use. See rights and permissions. Published by BMJ.

${ }^{1}$ School of Health Sciences, The University of Notre Dame Australia, Fremantle, Western Australia, Australia

${ }^{2}$ Institute for Health Research, The University of Notre Dame Australia, Fremantle, Western Australia, Australia

${ }^{3}$ Exercise Medicine Research Institute and School of Medical and Health Sciences, Edith Cowan University, Joondalup, Western Australia, Australia

Correspondence to Dr Sarah Ann Harris; Sarah.Harris@nd.edu.au

\section{ABSTRACT}

Objectives To explore the association between depressive symptoms and recent head-related trauma (diagnosed concussion, subconcussive impacts) in semiprofessional male Australian Football (AF) players. Methods Sixty-nine semiprofessional male players from a West Australian Football League (WAFL) club participated in the study ( $\mathrm{M}_{\text {age }}=21.81, \mathrm{SD}=2.91$ years). Depressive symptoms were measured using the Centre for Epidemiological Studies Depression Scale. Injuries and potential confounding variables (eg, pre-existing mental health condition; alcohol or drug hangovers; experiencing a stressful event) were self-reported anonymously using the WAFL Injury Report Survey. Both tools were administered every 2-weeks over the first 22-weeks of the WAFL season. Controlling for potential confounding variables and other injuries, a repeated measures generalised estimating equations model assessed the risk of clinically relevant depressive symptoms occurring, when diagnosed concussion or subconcussive impacts were experienced.

Results A total of 10 concussions and 183 subconcussive impacts were reported. Players who experienced a concussion were almost nine times more likely to experience clinically relevant depressive symptoms (OR 8.88, 95\% Cl 2.65 to 29.77, $\mathrm{p}<0.001$ ). Although elevated, depressive symptoms following subconcussive impacts were not statistically significant (OR $1.13,95 \%$ Cl 0.67 to $1.92, p=0.641$ ).

Conclusion These findings indicate that semiprofessional AF athletes may be at risk of experiencing depressive symptoms after concussion. Severity (concussion vs subconcussive impacts) and dose (number of impacts) appear to have an important relationship with depressive symptom outcomes in this cohort and should be considered for further research and management of player welfare.

\section{INTRODUCTION}

Mental health was recently elevated to an Australian National Health Research Priority area. ${ }^{1}$ Young adults (18-24 years) are experiencing mental illness at rates one-third higher than the average population, ${ }^{2}$ with suicide the leading cause of death for men aged 15-24

\section{What are the new findings}

- A greater number of injuries were reported using self-report methodology in comparison to the club medical team data.

- Recent diagnosed concussion was associated with a significant increased risk of experiencing clinically relevant depressive symptoms.

- Subconcussive impacts and other injuries were not significantly associated with an increase in depressive symptoms.

years in Australia. ${ }^{3}$ Semiprofessional ${ }^{4-6}$ and elite $^{7}$ Australian Football (AF) players are within this vulnerable age group. Yet, while there is increasing literature regarding the mental health of Australian elite athletes in general, ${ }^{8}$ there is a paucity of research pertaining in AF across all levels.

Depression is a common mental health illness which is both complex and multifaceted in nature. ${ }^{9}{ }^{10}$ Engagement in team sport may reduce the risk of an athlete experiencing depression ${ }^{11}$ because of the protective nature of social connection, ${ }^{12}$ social support ${ }^{13}$ and physical fitness. ${ }^{14}$ Conversely, athletes are at elevated risk of experiencing depressive symptoms ${ }^{15}$ due to sport-specific factors, including pressure to perform, ${ }^{13} 16$ musculoskeletal injury ${ }^{16}$ and, perhaps, concussion. ${ }^{1116}$

Despite an increase in concussion surveillance studies in elite $\mathrm{AF},{ }^{17-19}$ little is known about community or semiprofessional Western Australian players. While one study using head impact-sensory data indicated male semiprofessional West Australian Football League (WAFL) players may be exposed to more than 29 (SD=37) impacts per game, ${ }^{6}$ there are no other known WAFL concussion or subconcussive impact exposure or injury surveillance data. Exposure to head-related trauma is of concern, with numerous American athlete studies identifying concussion 


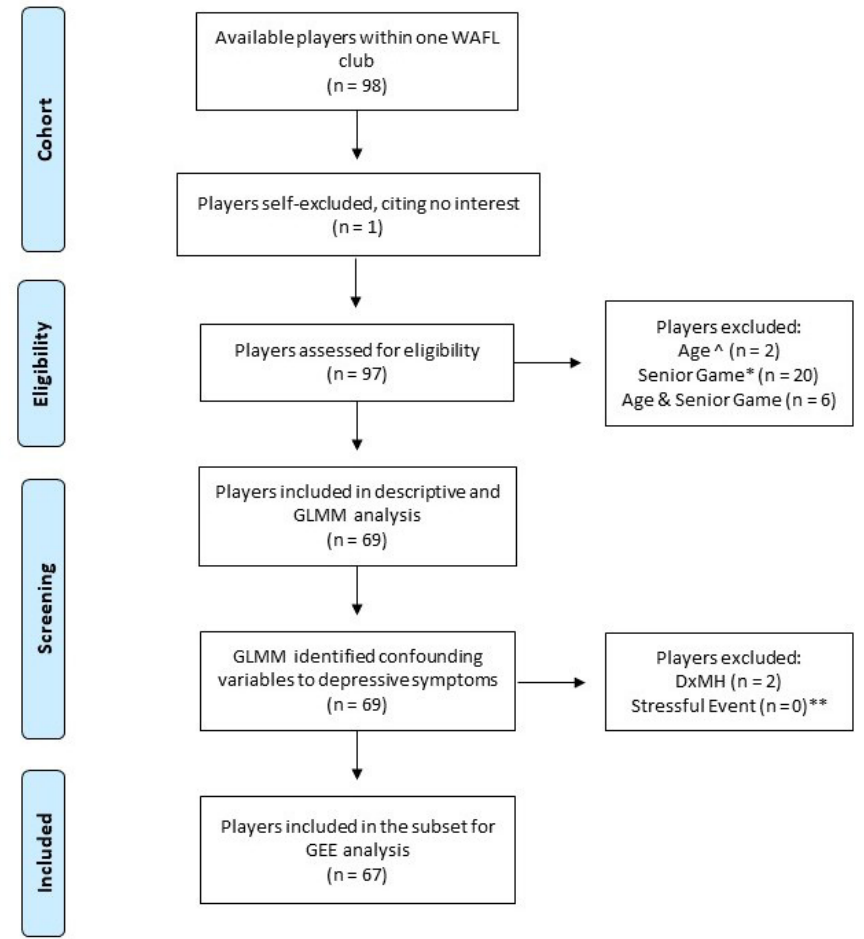

Figure 1 Participant eligibility and inclusion details for data analysis. Age: under 18 years of age as of the first data collection occasion. *Senior game: failed to report playing a minimum of one senior game (league or reserves) during the data collection period. ${ }^{*}$ Stressful event: players who reported a stressful event were excluded only for the corresponding occasion when they identified a stressful event had occurred. Given no players reported a stressful event for all occasions, no players were excluded from the total analysis. DxMH, diagnosed mental health; GEE, generalised estimating equation; GLMM, generalised linear mixed model; WAFL, West Australian Football League.

and repetitive subconcussive impacts ${ }^{20}$ to be associated with long-term health issues, including neurodegeneration and depressive symptoms in later life. ${ }^{21-23}$

Emerging research has explored the prevalence of depressive symptoms following recent concussion, ${ }^{24} 25$ predominately within American high school and collegiate athletes including football and rugby. ${ }^{162-32}$ Findings indicate depressive symptoms may be elevated up to 2-weeks after concussion ${ }^{28}$; however, it is unknown if these findings are applicable to AF. It is important for players and medical staff to consider the potential short-term psychological effects of concussion. ${ }^{1625}$

This can assist in earlier identification and referral to the appropriate care pathway allowing for treatment aimed at improving both a player's physical and mental welfare. Furthermore, despite the potential for subconcussive impacts to elicit similar long-term neurological sequelae as concussion, 223334 no studies to date have investigated whether recent subconcussive trauma evokes similar depressive symptom responses.

There is limited research exploring depressive symptoms or head-related trauma in semiprofessional AF players, and no known research has examined the association between these two domains in a male AF population. Given the majority of semiprofessional AF players are within the high-risk age category for mental ill health, ${ }^{4}$ and associations between concussion and depressive symptoms have been identified in other sports, 162930 the aims of this study were: (1) identify the prevalence of concussion and subconcussive impacts in male WAFL players over a competitive season; and (2) investigate associations between depressive symptoms and concussion or subconcussive impacts.

\section{METHODS}

\section{Study design and setting}

This prospective cohort study with repeated measures design used coded (deidentified), self-report survey methodology to gather injury, demographic and depressive symptom data over 22-weeks within the WAFL. The WAFL is a male semiprofessional league, which constitutes the second-tier competition for AF within Western Australia. Self-report methodology was employed because it is a simple, valid and reliable method to collect injury data in non-elite sporting environments. ${ }^{35}$ The Strengthening the Reporting of Observational Studies in Epidemiology checklist for longitudinal studies guided the reporting of this study (online supplementary file 1 ). ${ }^{36}$

\section{Participants}

Inclusion criteria included any male player aged 18 years and older who played at least one senior WAFL game during the 2015 season. Of the 98 senior players registered to the participating WAFL club, one player declined to participate citing 'no interest' and a further 28 individuals failed to meet the inclusion criteria due to age and minimum game requirements (figure 1). Therefore, 69 players were included. The sample size was calculated based on previous Centre for Epidemiologic Studies Depression Scale (CES-D) research ${ }^{32}$ with the probability (power) of $80 \%$ and the type I error probability $\alpha=0.05$. The a priori power calculation indicated a convenience sample of 50 participants over the age of 18 was adequate to examine the study hypothesis (online supplementary file 2).

\section{Survey development}

\section{WAFL Injury Report Survey}

The WAFL Injury Report Survey (WIRS) was developed by a panel of researchers and an industry representative following a review of previous literature and current injury and depressive symptom survey methodology in contact sport. ${ }^{1138}$ Face validity was established using five male WAFL players from different clubs, who reviewed the survey over three occasions. Formative review by both researchers and players, in conjunction with previous literature, ${ }^{35}$ indicated fortnightly survey intervals would elicit more accurate responses than fourweekly, and be less cumbersome than weekly. The modified WIRS was piloted with one WAFL team $(n=40)$ over two 
consecutive training sessions to assess test-retest reliability which demonstrated a strong level of agreement on demographic survey items (93\%-100\% agreement, $\mathrm{k}=0.82-1.00)$ and all injury variables $(95 \%-100 \%$ agreement, $\alpha=0.78-1.00)$.

Injury was defined as any injury or physical complaint sustained during a match or training, self-perceived by the player to cause pain; or impact, hinder or affect performance. ${ }^{39}$ Injury was classified as a self-reported subconcussive impact (head, neck or face), upper body injury (trunk, upper extremity), lower body injury (pelvis, lower extremities) or a medically diagnosed concussion (diagnosed by a medical practitioner). As the study focused on head-related injuries, for data analysis purposes, upper body and lower body injuries were grouped to form the variable other injury.

\section{Depressive symptoms}

Depressive symptoms are a facet of an individual's emotional well-being, and commonly present as negative feelings or thoughts including low mood, sleep disturbance or feelings of worthlessness. ${ }^{40}$ Although the presence of these symptoms does not infer a depression diagnosis, the number and severity of symptoms experienced at any one time could indicate a depressed mood. Depressive symptoms were measured fortnightly using the CES-D20. ${ }^{40}$ This 20 -item questionnaire is a reliable $(\alpha=0.85-0.90)$ screening tool for identifying depressive symptoms in the general population and with young adult males in sporting environments, ${ }^{8} 1116$ including postinjury and postconcussion. ${ }^{16}$ The tool demonstrates good sensitivity $(87 \%)$ and specificity $(77 \%) .{ }^{81141}$ Depressive symptom scores were calculated as total scores (sum score of the CES-D20 per occasion) with scores ranging from 0 to 60 , with an increased score indicating an increased presence of clinically relevant depressive symptoms. ${ }^{1140}{ }^{42}$ Consistent with common CES-D20 analysis procedures, ${ }^{11}{ }^{40-43}$ scores were dichotomised with a cutoff score of 16 or above indicating depressive symptoms are present. The CES-D20 shows moderate test-retest reliability over 2-weeks to 12 -months $(0.45-0.70)$ which is expected due to the natural fluctuation in symptoms. ${ }^{40} 41$ Given the multifactorial nature of depressive symptoms, ${ }^{9} 1025$ potential confounding variables to depressive symptom reporting were taken into consideration for analysis. These included a player's age ${ }^{11}{ }^{44}$ relationship status, change in relationship status, employment status, change in employment status, ${ }^{44}$ alcohol/other drug use, ${ }^{2545} 46$ illness, ${ }^{47} 48$ experiencing a recent stressful event (stressful event $)^{25}$ and reporting a pre-existing diagnosed mental health (DxMH) condition. ${ }^{11} 294449$

\section{Survey procedures}

Players completed the WIRS and CES-D20 every 2-weeks (fortnight), post-training on the same night for 22-weeks of the regular playing season (11 data collection occasions). Players recalled total injuries received per injury category during the game and each training session every fortnight. Within the fortnightly survey period, a player would normally participate in up to six evening training sessions and two games (one per weekend). A unique identification code enabled participant's responses to be tracked over time while ensuring responses were coded.

\section{Additional data}

Anonymous medical team data (MTD) were also provided by the club, as a sum of each injury type per week for the corresponding 22-week period of time. Using the same four injury classifications as the WIRS, the MTD were based on the sum of injuries reported to the club physiotherapist, doctor or head trainer during training or competition. No further information was provided to the research team.

\section{Statistical analysis}

SPSS V.24.0 was used for all statistical analysis. Repeated measures non-uniform Bland-Altman limits of agreement analysis was used to assess the level of agreement (95\% limits $)^{50}$ between total sum of self-reported WIRS and MTD injuries. Injury prevalence was calculated based on the number of participants who reported the injury type, divided by the total sample population. Injury rate was also presented per frequency of athlete exposures $(\mathrm{AE})$, where one match or one practice per person was considered one $\mathrm{AE} .{ }^{51-53} \mathrm{AE}$ rates are challenging to calculate at a non-elite level, as players may not always attend all training sessions. ${ }^{54}$ Due to the study parameters, a conservative AE estimate was used, where the total number of sessions was calculated based on the standard six training sessions and two games per occasion, taking into consideration any scheduled byes (ie, no games), and the number of responses per occasion for the study period. The $\mathrm{AE}$ rates were calculated based on the sum of injuries divided by the number of AEs, and presented per $1000 \mathrm{AEs}^{5153}$ with $95 \% \mathrm{CI}^{53}$ :

$$
\begin{aligned}
\text { AE Rate } & =\frac{\sum \text { Number of Injuries }}{\sum \text { Athlete }- \text { Exposures }} \times 1000 \\
95 \% \mathrm{CI}= & \left(\left(\frac{\sum \text { Number of Injuries }}{\sum \text { Athlete }- \text { Exposures }}\right) \pm\right. \\
& \left(1.96 \times \frac{\sqrt{\text { Number of Injuries }}}{\sum \text { Athlete }- \text { Exposures }}\right) \times 1000
\end{aligned}
$$

An independent t-test (non-parametric alternative Mann-Whitney $U$ test) was used to assess differences in baseline depressive symptom scores between players who did or did not receive concussion during the season.

A two-stage regression modelling strategy was used to account for the multifactorial nature of depressive symptoms. ${ }^{9}$ First, in a generalised linear mixed model (GLMM), with normal probability distribution, identity link function was used to investigate the longitudinal association between depressive symptoms (CES-D20 scores) and nine potential confounding variables. A GLMM was used as it allows for longitudinal repeated measures data with multiple independent variables. ${ }^{55}$ These confounders included: age 
Table 1 WIRS fortnightly response rate over the 22-week playing season $(n=69)$

$\begin{array}{llllllllllll}\text { Occasion } & 1 & 2 & 3 & 4 & 5 & 6 & 7 & 8 & 9 & 10 & 11\end{array}$

$\begin{array}{llllllllllll}\text { Responses } & 59 & 60 & 52 & 58 & 55 & 57 & 56 & 56 & 58 & 51 & 52\end{array}$

$\begin{array}{llllllllllll}\text { Missing }^{*} & 10 & 9 & 17 & 11 & 14 & 12 & 13 & 13 & 11 & 18 & 17\end{array}$

Data were collected during the playing season on the same night, each fortnight (11 occasions). Therefore, each occasion reflects a 2-week period.

*Not all participants responded on every occasion. Due to the deidentified nature of the study, if a player missed an occasion, a response was unable to be captured. A total of 614 responses were captured.

WIRS, WAFL Injury Report Survey.

(continuous variable, measured as a constant), relationship status, change in relationship status, employment status, change in employment status, alcohol/other drug use, illness, stressful event and pre-existing DxMH condition (dichotomous variables measured per occasion). These confounders were examined within the same model to reduce the risk of bias. ${ }^{56}$ Additionally, quasilikelihood under independence model criterion (QIC) was examined to determine the model fit, where the lower the value the better the model fit. ${ }^{57}$

Second, a generalised estimating equation (GEE) model for dichotomous outcome modelled the risk of depressive symptoms, using the binary cut-off (CES-D20 $\geq 16$ ) 1140424358 when an injury (concussion, subconcussive impact or other injury measured as continuous variables) was reported on each occasion. Multiple strengths of the GEE include: accounting for individual's repeated measures data over time ${ }^{5960}$ not relying on specific time points and accounting for missing data. ${ }^{59}$ This model enables concussion and subconcussive impacts to be compared, while controlling for other injuries. This method has been used in other AF injury risk studies ${ }^{76162}$ and to explore depressive symptoms and general injury in college athletes. ${ }^{32} 5860$ The level of statistical significance was set at $\mathrm{p}<0.05$.

\section{Patient and public involvement}

Patients and/or the public were involved in the design, or conduct, or reporting, or dissemination plans of this research.

\section{RESULTS}

\section{Injury prevalence}

Players $(n=69)$ were on average 21.9 years $(\mathrm{SD}=2.96$, range: $18-31$ years), and completed 8.90 data collection occasions $(\mathrm{SD}=3.30)$ with $48 \%$ completing all 11 occasions $(\mathrm{n}=33)$ (table 1$)$.

The sample had a concussion prevalence of $13 \%(n=9)$, with a total of 12 concussions reported by nine players during the data collection period. A subconcussive impact prevalence of $51 \%(n=35)$ was recorded (table 2$)$. A total of 14 players $(20 \%)$ reported no injuries at all over the data collection period.

A total of 495 injuries were reported in the WIRS. Comparatively, MTD indicated a total of 175 injuries across the same period of time with the same playing cohort. Although the WIRS identified a greater sum of injuries than the MTD, the Bland-Altman analysis found the two measures had a good level of agreement as the majority of points were positioned within the $95 \% \mathrm{CI}$ limits of agreement (online supplementary file 3). This indicates both injury reports were proportionally measuring the same outcome.

\section{Confounding variables to depressive symptom reporting}

A GLMM confirmed a significant association between DxMH and CES-D20 scores $(\beta=10.02,95 \%$ CI 2.52 to 17.53, $\mathrm{p}=0.009)$. Therefore, participants who identified DxMH on the first data collection occasion were excluded from all further analyses as per the exclusion criteria for the study. The GLMM confirmed that stressful event $(\beta=2.72$, $95 \%$ CI 1.29 to $4.15, \mathrm{p}<0.001)$ also significantly increased CES-D20 scores, hence, to control for this influence, data from participants who reported a stressful event were excluded for the corresponding occasion when they identified injury had occurred (figure 1). The remaining seven demographic variables were not significantly associated with depressive symptoms ( $p>0.287)$. Model QICs were examined with all nine confounders $(\mathrm{n}=69$, $\mathrm{QIC}=372.420)$, seven confounders (excluding DxMH and stressful event, $\mathrm{n}=67, \mathrm{QIC}=295.222$ ) and the final model (inclusion of injury variables and no adjustment for confounders, $n=67$, QIC=280.816). The final injury model without adjustment for confounders provided the best model fit.

Table 2 Prevalence and rate of self-reported injuries over the 11 occasions $(n=69)$

\begin{tabular}{lccccc}
\hline & Participants (n) & Injury sum & Prevalence (\%) & AE & \multicolumn{1}{c}{ AE 95\% Cl } \\
\hline Concussion & 9 & 12 & 13 & 2.56 & 1.11 to 4.01 \\
Subconcussive impacts & 35 & 194 & 51 & 41.36 & 35.54 to 47.19 \\
Upper body & 38 & 132 & 55 & 28.14 & 23.34 to 32.95 \\
Lower body & 46 & 157 & 67 & 33.48 & 28.24 to 38.71 \\
Total injuries & 55 & 495 & 80 & 105.54 & 96.25 to 114.84 \\
\hline
\end{tabular}

Estimated total athlete exposures: 4690.

AE: estimated rate per 1000 athlete exposures, where an $A E$ is defined as one player participating in one match or one practice.

$A E$, athlete exposure. 
Table 3 Mean depressive symptom scores (CES-D20) based on the number of subconcussive impacts reported per occasion*

\begin{tabular}{lrrl}
\hline $\begin{array}{l}\text { Total } \\
\text { subconcussive } \\
\text { impacts per }\end{array}$ & & \multicolumn{2}{c}{ Depressive symptoms $\dagger$} \\
\cline { 2 - 4 } occasion & $\mathbf{n}$ & Mean & SD \\
\hline 0 & 407 & 7.34 & 5.53 \\
1 & 48 & 7.90 & 6.16 \\
2 & 33 & 7.49 & 5.29 \\
3 & 9 & 9.00 & 5.36 \\
4 & 4 & 11.00 & 10.00 \\
5 & 1 & 15.00 & $\ddagger$ \\
\hline
\end{tabular}

A dose-response relationship for concussion per player was unable to be explored, as a maximum of one concussion was reported per occasion.

*Excluded participants who had reported a stressful event or diagnosed mental health (DxMH), or incomplete CES-D20 (total sample included $n=67$ ); total of 502 cases were included from 67 participants over 11 occasions.

†Depressive symptoms is the CES-D20 sum score. CES-D20 $\geq 16$ denotes presence of clinically relevant depressive symptoms. $\ddagger S D$ unable to be calculated due to sample size $(n=1)$.

CES-D20, Centre for Epidemiologic Studies Depression Scale.

\section{Depressive symptoms and head-related trauma}

Based on these exclusion criteria, 10 incidences of concussion and 183 subconcussive impacts were analysed. Shapiro-Wilk analysis indicated that total CES-D20 scores were not normally distributed $(\mathrm{p}<0.001)$. At baseline, although elevated, there was no significant difference $(\mathrm{p}=0.287)$ between depressive symptom scores for athletes who experienced a concussion $(\mathrm{M}=9.69$, $\mathrm{SD}=3.81, \mathrm{Mdn}=9.00$ ), compared with athletes who did not experience a concussion during the testing period $(\mathrm{M}=7.04, \mathrm{SD}=5.57, \mathrm{Mdn}=6.50)$.

A higher depressive symptom score was reported following concussion $(\mathrm{M}=12.90, \mathrm{SD}=7.36)$ compared with subconcussive impact/s per occasion $(\mathrm{M}=8.06, \mathrm{SD}=5.94)$. However, experiencing any head-related trauma elicited greater mean depressive symptom scores in comparison to other injuries $(\mathrm{M}=7.62, \mathrm{SD}=5.92)$, or no injuries $(\mathrm{M}=7.56, \mathrm{SD}=5.51)$.

Four out of 10 concussive episodes (40\%) were associated with players experiencing clinically relevant depressive symptoms (CES-D20 $\geq 16$ ). In comparison, only $8 \%$ of players with a minimum of one subconcussive impact, $8 \%$ of players who recalled no injuries and $7 \%$ of players who reported other injuries reported clinically relevant depressive symptoms. Descriptively, a doseresponse relationship with a greater depressive symptom score was observed, when multiple subconcussive impacts were reported per occasion (table 3).

\section{Risk of depressive symptoms following head-related trauma}

The GEE model indicated players who reported a concussion were almost nine times more likely to experience clinically relevant depressive symptoms within a 2-week period (table 4). Controlling for all other injuries including concussion, while not statistically significant, the GEE OR identified subconcussive impacts increased the probability of clinically relevant depressive symptoms by $13 \%$ per impact reported (table 4 ). Other injuries per occasion were not significantly associated with depressive symptoms.

\section{DISCUSSION}

To our knowledge, this is the first study to longitudinally explore depressive symptoms and recent head-related trauma in WAFL players over a competitive season. We present the first injury surveillance data on concussion and subconcussive impacts within WAFL and found participants who reported diagnosed concussion, reported clinically relevant depressive symptoms at higher rates than previously reported in athletes in other sports.

\section{Injury prevalence}

Our results identified a greater number of concussions (10 concussions per 67 players) than Victorian Community AF (2.23 concussions per team/season) or elite AF (1.5 concussions, per club of approximately 45 players) ${ }^{63}{ }^{64}$ although methodological differences to previous AF studies are noted. Novel to previous findings,

Table 4 GEE model: repeated measures assessment of risk of depressive symptoms* following injury

\begin{tabular}{|c|c|c|c|c|c|}
\hline Parameter & $\beta$ & SE & OR & $\begin{array}{l}\text { OR } \\
95 \% \mathrm{Cl}\end{array}$ & $P$ value \\
\hline Intercept & -2.51 & 0.28 & 0.08 & 0.05 to 0.14 & $<0.001$ \\
\hline Diagnosed concussion & 2.18 & 0.62 & 8.878 & 2.65 to 29.77 & $<0.001 \dagger$ \\
\hline Subconcussive impact & 0.13 & 0.27 & $1.133 \ddagger$ & 0.67 to 1.92 & 0.641 \\
\hline Other injury & -0.17 & 0.23 & 0.840 & 0.53 to 1.32 & 0.451 \\
\hline
\end{tabular}

*Depressive symptoms assessed as a binary variable, with Centre for Epidemiologic Studies Depression Scale (CES-D20) sum score $\geq 16$, indicating presence of clinically relevant depressive symptoms.

†Bold values denote statistical significance at $p<0.05$; OR derived from $\operatorname{Exp}(\beta)$. OR $>1.00$ indicates an increase in the risk of depression. ‡Despite a non-significant association, OR $(\operatorname{Exp} \beta)$ denotes an increase in depressive symptoms, per subconcussive impact reported.

GEE, generalised estimating equation. 
the 67 players involved in our study reported 183 subconcussive impacts over the season which are not commonly formally reported as an injury unless they cause facial fractures or a player misses a game. ${ }^{38}$ Given the substantial number of subconcussive impacts reported and that concussion is difficult to diagnose ${ }^{65}$ it may be advisable for future injury reporting systems to include a category to specifically capture subconcussive impacts.

We demonstrated self-report questionnaires can successfully gather injury data in semiprofessional AF. Strong agreement was found between the WIRS findings and the club's medical records, although the number of injuries captured by the WIRS was larger. This is consistent with self-report research ${ }^{66} 67$ which suggests players underreport their injuries, particularly head trauma, to medical staff. Perhaps the WIRS coded self-report nature, reduced a player's hesitation to disclose injury information; however, further research is recommended to explore potential reasons and differences in reporting within this cohort.

\section{Association between depressive symptoms and head-related trauma}

The second aim of our study was to explore whether concussion and subconcussive impacts were associated with an increase in a player's self-reported depressive symptoms within the 2-week period of being reported. The prevalence of depression in the Australian young male adult population is estimated to be $12 \%{ }^{68}$ Although the CES-D20 does not diagnose depression, only $8 \%$ of players who did not report an injury, reported depressive symptom scores of clinical relevance. Though a direct comparison cannot be made, this may indicate that in the current non-injured sample, depressive symptoms were less prevalent than in the general population for young male Australian adults.

In contrast, following concussion, a greater proportion of players reported clinically relevant depressive symptoms $(40 \%)$. This is in comparison to baseline, population prevalence and when compared with American college athletes $(4 \%-14 \%$; using CES-D20 or other measures). ${ }^{1628}$ The reason for these discrepancies is unclear; however, it may be due to methodological differences in data collection, including the different measures to assess depressive symptoms, the smaller number of concussions available for analysis, the level of competition, the type of contact sport, game demands or other personal predisposing factors.

Even if a player is not diagnosed with a concussion, their brain may still be exposed to external forces resulting from subconcussive impacts. ${ }^{3469} 70$ Although descriptive statistics identified a dose-response relationship between multiple subconcussive impacts and an increase in depressive symptom score, the GEE analysis did not find a significant association with depressive symptom scores of clinical relevance. Subconcussive impacts and concussion may be inter-related, therefore providing a colinear relationship, further explaining the non-significant findings. Despite the emerging evidence of long-term outcomes including emotional changes and neurodegeneration ${ }^{21-23}$ following subconcussive impacts ${ }^{3469} 70$ perhaps, short-term changes in depressive symptoms following these impacts are not present or not detectable in this population. Alternatively, future research should consider if the intensity or magnitude of the impact, or multiple impacts in quick succession influences depressive symptoms.

In previous musculoskeletal injury studies, an increase in depressive symptoms was attributed to exclusion from play, ${ }^{71}$ however, specifically in concussed players, other literature indicated depressive symptoms were shown to decrease with time, regardless of participation or exclusion status. ${ }^{16}{ }^{30}$ While these elements were not explored in the current cohort, our findings identified that recent other injuries were not associated with increases in depressive symptoms. Therefore, perhaps it is the impact, rather than the exclusion from play, that contributes to increases in depressive symptoms in concussed athletes.

\section{Implications for practice}

Access to medical resources declines as the level of professionalism decreases, hence self-report may assist non-elite clubs to capture reliable injury information. ${ }^{72}$ Although the association between recent concussion and increases in depressive symptoms needs further validation, given the potential risks associated with depressive states, clubs and coaches may need to consider these findings to ensure the well-being of their players. With the focus during concussion recovery often based on somatic symptoms and return-to-play strategies, emotional assessment may be required to identify those players exhibiting depressive symptoms. Given many players do not associate emotional changes (ie, depressive symptoms) with receiving head trauma, ${ }^{73}$ further education and access to professional resources for players and staff is required.

\section{Strengths and limitations}

This study has a number of strengths. Engaging the entire club playing cohort likely reduced the risk of respondent bias. Additionally, maintaining player anonymity may have increased reporting compliance and reduced the risk of inaccurate reporting. ${ }^{66}$ Furthermore, the longitudinal design of the study strengthens the rigour of the findings as players were tracked over the duration of the season.

Regarding limitations, assessing emotional response to injury presents numerous methodological difficulties. A primary limitation of this research involves the 2-week collection of injury and depressive symptom data because an injury may have occurred at any point within the 2-week period, therefore the days after injury may differ between players. Future research may explore how depressive symptoms fluctuate in the number of days after concussive or subconcussive impact (0-14days) and implications to optimise follow-up. A secondary limitation relates to the generalisability of these findings. This study was limited to one semiprofessional WAFL club, therefore the generalisability of results to other levels is unknown. Due to the low number of concussions reported, and the three injury groups, it is likely that this analysis may be underpowered. 
Issues with sample size may be addressed in future research by considering the use of penalised GEE analysis in alternate statistical packages where this procedure is available. Third, the self-report nature of the study means the WIRS relies on accurate and honest reporting from players, although the WIRS validation and test-retest reliability suggests the responses were accurate. Ideally, data would be collected using medical and allied health practitioner reports to ensure diagnostic accuracy. However, a semiprofessional environment, relying on medical teams to collect accurate and reliable injury data, can be difficult for numerous reasons. Players may choose to withhold injury information from medical staff for fear of being withheld from competition, not recognising that an injury has occurred, or not perceiving the injury as serious enough to warrant reporting. ${ }^{6674}$ Under-reporting can be compounded at a semiprofessional and community level, due to reduced access to qualified medical staff. ${ }^{72}$ Therefore, coded selfreport methodology may overcome some of these barriers and elicit greater compliance in injury and concussion reporting. ${ }^{66}$ Finally, while effort was made to control for additional factors which may influence the presence of depressive symptoms, due to the multifactorial nature of mental health, other unmeasured factors may have influenced the findings.

\section{CONCLUSION}

Often the focus in AF is based around performance or physical health, yet our research considered an aspect of mental health. Specifically, it is the first study to consider the effect of recent concussion and subconcussive impacts on an AF player's depressive symptoms. In summary, WAFL players may elicit an increase in depressive symptoms, following recent exposure to concussion. However, it appears subconcussive impacts do not significantly elicit the same emotional response. Recognising $\mathrm{AF}$ players may experience emotional changes following concussion, can assist medical staff and coaches with providing education and ongoing care for players, thereby improving the well-being of their players.

\section{Twitter Sarah Ann Harris @DrSarahHarris}

Acknowledgements The authors acknowledge Dr Lauren Fortington (Australian Centre for Research into Injury in Sport and its Prevention, ACRISP) for her revision suggestions and the reviewers for their valuable comments. In addition, the authors thank the research participants for their time and the WAFL staff members who agreed to involvement in the research.

Contributors All authors made substantial contribution to the material submitted including study design, interpretation of results and manuscript development and review. SAH completed the data collection, statistical analysis and manuscript writing. PTC and MB consulted on the statistical analysis.

Funding SAH was supported by an Australian University Postgraduate Award (UPA) scholarship.

\section{Competing interests None declared.}

Patient consent for publication Not required.

Ethics approval The University of Notre Dame Australia's Human Research Ethics Committee (014049F).

Provenance and peer review Not commissioned.

Data availability statement № data are available. № additional data available.
Open access This is an open access article distributed in accordance with the Creative Commons Attribution Non Commercial (CC BY-NC 4.0) license, which permits others to distribute, remix, adapt, build upon this work non-commercially, and license their derivative works on different terms, provided the original work is properly cited, appropriate credit is given, any changes made indicated, and the use is non-commercial. See: http://creativecommons.org/licenses/by-nc/4.0/.

ORCID iD

Sarah Ann Harris http://orcid.org/0000-0003-0240-4386

\section{REFERENCES}

1 NHMRC. Mental health research Advisory Committee, 2018. Available: https://nhmrc.gov.au/mental-health-research-advisorycommittee [Accessed 8 Jan 2019].

2 Department of Health and Ageing. National mental health report 2013: tracking progress of mental health reform in Australia, 19932011. Canberra, 2013. www.health.gov.au/mentalhealth

3 Australian Institute of Health and Welfare. Australia's health 2018. Canberra, 2018.

4 West Australian Football Commission Inc. WAFL player profiles, 2014. Available: www.wafl.com.au [Accessed 23 Jun 2014].

5 Pearce AJ, Hoy K, Rogers MA, et al. Acute motor, neurocognitive and neurophysiological change following concussion injury in Australian amateur football. A prospective multimodal investigation. J Sci Med Sport 2015;18:500-6.

6 King D, Hecimovich M, Clark T, et al. Measurement of the head impacts in a sub-elite Australian rules football team with an instrumented patch: an exploratory analysis. Int J Sports Sci Coach 2017;12:359-70.

7 Stares J, Dawson B, Peeling P, et al. Identifying high risk loading conditions for in-season injury in elite Australian football players. $J$ Sci Med Sport 2018;21:46-51.

8 Gulliver A, Griffiths KM, Mackinnon A, et al. The mental health of Australian elite athletes. J Sci Med Sport 2015;18:255-61.

9 Dodge R, Daly A, Huyton J, et al. The challenge of defining wellbeing. Intnl J Wellbeing 2013;2:222-35.

10 Hopwood MJ, Malhi G. To screen for depression or not? Med J Aust 2016;204:329-30.

11 Yang J, Peek-Asa C, Corlette JD, et al. Prevalence of and risk factors associated with symptoms of depression in competitive collegiate student athletes. Clin J Sport Med 2007;17:481-7.

12 Pierce D, Liaw S-T, Dobell J, et al. Australian rural football Club leaders as mental health advocates: an investigation of the impact of the coach the coach project. Int J Ment Health Syst 2010;4:10-17.

13 Nixdorf I, Frank R, Beckmann J. Comparison of athletes' proneness to depressive symptoms in individual and team sports: Research on psychological mediators in junior elite athletes. Front Psychol 2016;7:1-8.

14 Brown DR, Galuska DA, Zhang J, et al. Psychobiology and behavioral strategies. physical activity, sport participation, and suicidal behavior: U.S. high school students. Med Sci Sport Exerc 2007;39:2248-57.

15 Gorczynski PF, Coyle M, Gibson K. Depressive symptoms in high-performance athletes and non-athletes: a comparative metaanalysis. Br J Sports Med 2017;51:1348-54.

16 Roiger T, Weidauer L, Kern B. A longitudinal pilot study of depressive symptoms in concussed and injured/nonconcussed national collegiate athletic association division I student-athletes. J Athl Train 2015;50:256-61.

17 Makdissi M, Davis G. Using video analysis for concussion surveillance in Australian football. J Sci Med Sport 2016;19:958-63.

18 McIntosh AS, Patton DA, Fréchède B, et al. The biomechanics of concussion in unhelmeted football players in Australia: a casecontrol study. BMJ Open 2014;4:e005078-9.

19 Gibbs N, Watsford M. Concussion incidence and recurrence in professional Australian football match-play: a 14-year analysis. $J$ Sports Med 2017;2017:1-7.

20 Gavett BE, Stern RA, McKee AC. Chronic traumatic encephalopathy: a potential late effect of sport-related concussive and subconcussive head trauma. Clin Sports Med 2011;30:179-88.

21 Kerr ZY, Marshall SW, Harding HP, et al. Nine-Year risk of depression diagnosis increases with increasing self-reported concussions in retired professional football players. Am J Sports Med 2012;40:2206-12.

22 Montenigro PH, Alosco ML, Martin BM, et al. Cumulative head impact exposure predicts later-life depression, apathy, executive dysfunction, and cognitive impairment in former high school and College football players. J Neurotrauma 2017;34:328-40. 
23 Guskiewicz KM, Marshall SW, Bailes J, et al. Association between recurrent concussion and late-life cognitive impairment in retired professional football players. Neurosurgery 2005;57:719-26.

24 Yrondi A, Brauge D, LeMen J, et al. Depression and sportsrelated concussion: a systematic review. La Presse Médicale 2017; 46:890-902.

25 Rice SM, Parker AG, Rosenbaum S, et al. Sport-Related concussion and mental health outcomes in elite athletes: a systematic review. Sports Med 2018;48:447-65.

26 Meier TB, Bellgowan PSF, Mayer AR. Longitudinal assessment of local and global functional connectivity following sports-related concussion. Brain Imaging Behav 2017:11:129-40.

27 Meier TB, Bellgowan PSF, Singh R, et al. Recovery of cerebral blood flow following sports-related concussion. JAMA Neurol 2015;72:530-8.

28 Kontos AP, Covassin T, Elbin RJ, et al. Depression and neurocognitive performance after concussion among male and female high school and collegiate athletes. Arch Phys Med Rehabil 2012;93:1751-6.

29 Vargas G, Rabinowitz A, Meyer J, et al. Predictors and prevalence of postconcussion depression symptoms in collegiate athletes. J Athl Train 2015;50:250-5.

30 Mainwaring LM, Hutchison M, Bisschop SM, et al. Emotional response to sport concussion compared to ACL injury. Brain Inj 2010;24:589-97.

31 Singh R, Savitz J, Teague TK, et al. Mood symptoms correlate with kynurenine pathway metabolites following sports-related concussion. J Neurol Neurosurg Psychiatry 2016;87:670-5.

32 Yang J, Peek-Asa C, Covassin T, et al. Post-concussion symptoms of depression and anxiety in division I collegiate athletes. Dev Neuropsychol 2015;40:18-23.

33 Greenwald RM, Chu JJ, Beckwith JG, et al. A proposed method to reduce underreporting of brain injury in sports. Clin J Sport Med 2012;22:83-5.

34 Talavage TM, Nauman EA, Breedlove EL, et al. Functionally-detected cognitive impairment in high school football players without clinically-diagnosed concussion. J Neurotrauma 2014;31:327-38.

35 Valuri G, Stevenson M, Finch C, et al. The validity of a four week self-recall of sports injuries. Inj Prev 2005;11:135-7.

36 von Elm E, Altman DG, Egger M, et al. The strengthening the reporting of observational studies in epidemiology (STROBE) statement: guidelines for reporting observational studies. Int J Surg 2014;12:1495-9.

37 Armstrong S, Oomen-Early J, connectedness S. Social connectedness, self-esteem, and depression symptomatology among collegiate athletes versus nonathletes. J Am Coll Health 2009;57:521-6.

38 Orchard J, Seward H, Orchard JJ. 2014 AFL injury report. Melbourne, 2015. Available: http://www.afl.com.au/staticfile/AFL Tenant/AFL/Files/2014-AFL-Injury-Report.pdf [Accessed 9 Aug 2015].

39 Fuller CW, Ekstrand J, Junge A, et al. Consensus statement on injury definitions and data collection procedures in studies of football (soccer) injuries. Br J Sports Med 2006;40:193-201.

40 Radloff LS. A self-report depression scale for research in the general population. Appl Psychol Meas 1977:1:385-401.

41 Fisher C. Center for epidemiologic studies depression scale (CES-D): an excellent free psychological screening instrument for major depression., 2009. Available: http://www.bmedreport.com/archives/ 7139 [Accessed 25 May 2016].

42 Wolanin A, Hong E, Marks D, et al. Prevalence of clinically elevated depressive symptoms in college athletes and differences by gender and sport. Br J Sports Med 2016;50:167-71.

43 Pryor J, Larson A, DeBeliso M. The prevalence of depression and concussions in a sample of active North American semi-professional and professional football players. J Lifestyle Med 2016;6:7-15.

44 Andrews G, Hall W, Teesson M, et al. The mental health of Australians. Canberra, 1999. www.health.gov.au/hsdd/mentalhe

45 Putukian M. The psychological response to injury in student athletes: a narrative review with a focus on mental health. $\mathrm{Br} J$ Sports Med 2016;50:145-8.

46 Piccinelli M, Wilkinson G. Gender differences in depression: critical review. Br J Psychiatry 1999;177:486-93.

47 Coughlin SS. Anxiety and depression: linkages with viral diseases. Public Health Rev 2012;34:1-13.

48 Blank T, Detje CN, Spieß A, et al. Brain Endothelial- and epithelialspecific interferon receptor chain 1 drives virus-induced sickness behavior and cognitive impairment. Immunity 2016;44:901-12.
49 Du Preez EJ, Graham KS, Gan TY, et al. Depression, anxiety, and alcohol use in elite rugby League players over a competitive season. Clin J Sport Med 2017;27:530-5.

50 Myles PS, Cui J. Using the Bland-Altman method to measure agreement with repeated measures. Br J Anaesth 2007;99:309-11.

51 Kerr ZY, Roos KG, Djoko A, et al. Epidemiologic measures for quantifying the incidence of concussion in national collegiate athletic association sports. J Athl Train 2017;52:167-74.

52 Marshall SW, Guskiewicz KM, Shankar V, et al. Epidemiology of sports-related concussion in seven us high school and collegiate sports. Inj Epidemiol 2015;2:1-10.

53 Knowles SB, Marshall SW, Guskiewicz KM. Issues in estimating risks and rates in sports injury research. $J$ Athl Train 2006;41:207-15.

54 Finch CF, Diamantopoulou K, Twomey DM, et al. The reach and adoption of a coach-led exercise training programme in community football. Br J Sports Med 2014;48:718-23.

55 Müller S, Scealy JL, Welsh AH. Model selection in linear mixed models. Statist. Sci. 2013;28:135-67.

56 VanderWeele TJ. Principles of confounder selection. Eur J Epidemiol 2019;34:211-9.

57 Heinze G, Wallisch C, Dunkler D. Variable selection - A review and recommendations for the practicing statistician. Biom $J$ 2018:60:431-49.

$58 \mathrm{Li} \mathrm{H}$, Moreland JJ, Peek-Asa C, et al. Preseason anxiety and depressive symptoms and prospective injury risk in collegiate athletes. Am J Sports Med 2017;45:2148-55.

59 Bell ML, Fairclough DL. Practical and statistical issues in missing data for longitudinal patient-reported outcomes. Stat Methods Med Res 2014;23:440-59.

60 Yang J, Schaefer JT, Zhang N, et al. Social support from the athletic trainer and symptoms of depression and anxiety at return to play. $J$ Athl Train 2014;49:773-9.

61 Colby MJ, Dawson B, Peeling P, et al. Multivariate modelling of subjective and objective monitoring data improve the detection of non-contact injury risk in elite Australian footballers. J Sci Med Sport 2017;20:1068-74.

62 Orchard JW, Driscoll T, Seward H, et al. Relationship between interchange usage and risk of hamstring injuries in the Australian football League. J Sci Med Sport 2012;15:201-6.

63 Afl doctors association, AFL physiotherapists association, AFL football operations department. 2015 AFL injury survey. Melbourne, 2016. Available: www.afl.com.au/staticfile/AFLTenant/AFL/Files/ 2015-AFL-Injury-Report.pdf [Accessed 18 May 2017].

64 Ekegren CL, Gabbe BJ, Donaldson A, et al. Injuries in communitylevel Australian football: results from a club-based injury surveillance system. J Sci Med Sport 2015;18:651-5.

65 McCrory PR, Meeuwisse WH, Aubry M, et al. Consensus statement on concussion in sport: the 4th International Conference on concussion in sport held in Zurick, November 2012. Br J Sports Med;2013:250-8.

66 McCrea M, Hammeke T, Olsen G, et al. Unreported concussion in high school football players: implications for prevention. Clin J Sport Med 2004;14:13-17.

67 Meier TB, Brummel BJ, Singh R, et al. The underreporting of selfreported symptoms following sports-related concussion. J Sci Med Sport 2015;18:507-11.

68 Beyond Blue. Depression in males, 2016. Available: https://www. beyondblue.org.au [Accessed 6 Apr 2016].

69 Broglio SP, Eckner JT, Martini D, et al. Cumulative head impact burden in high school football. J Neurotrauma 2011;28:2069-78.

70 Shultz SR, MacFabe DF, Foley KA, et al. Sub-concussive brain injury in the Long-Evans rat induces acute neuroinflammation in the absence of behavioral impairments. Behav Brain Res 2012;229:145-52.

71 Ruddock-Hudson M, O'Halloran P, Murphy G. The psychological impact of long-term injury on Australian football League players. $J$ Appl Sport Psychol 2014;26:377-94.

72 Gilbert F, Partridge BJ. The need to tackle concussion in Australian football codes. Med J Aust 2012;196:561-3.

73 Chrisman SP, Quitiquit C, Rivara FP. Qualitative study of barriers to concussive symptom reporting in high school athletics. J Adolesc Health 2013;52:330-5.

74 Pearce AJ, Young JA, Parrington L, et al. Do as I say: contradicting beliefs and attitudes towards sports concussion in Australia. $J$ Sports Sci 2017;35:1911-9. 